\title{
Validation of Verdura intense moisturizing lotion for its moisturization benefits
}

\section{Gatla Venkata Amruthavalli, Vadivel Aruna, Rajagopal Gayathri}

\author{
Dr. JRK's Research and Pharmaceuticals Pvt. Ltd., Kunrathur, Chennai-600069, India
}

Corresponding author: Dr. Gatla Venkata Amruthavalli, E-mail: amrutha.valli4@gmail.com/research@jrkresearch.com

\begin{abstract}
Every skin needs moisturization and choosing right moisturizer is very important. No matter what kind of the skin one have, moisturizing it is required. In the current study we have evaluated the moisturizing ability of Verdura intense moisturizing lotion in human volunteers. Dermascope and moisture analyzer was used to know the texture of skin and moisture level respectively. The study shows the intense \& prolonged moisturization is achieved with the Verdura intense moisturizing lotion.
\end{abstract}

Key words: Moisturizing lotion, Analysis of moisturizer, Verdura intense moisturizing lotion

\section{INTRODUCTION}

Moisturizing of skin is very vital for healthy skin. Understanding the requirement of moisturization of skin regularly over the past decade, great progress has been made toward elucidating the structure and function of the stratum corneum (SC), the outermost layer of the epidermis [1].

$\mathrm{SC}$ cells (corneocytes) protect against desiccation and environmental challenge by regulating water flux and retention. Maintenance of an optimal level of hydration by the SC is largely dependent on several factors. Using of right moisturizers can balance both the moisturization and emolliency of the SC cells which gives healthy skin.

A good moisturizer should be able to provide moisturization to skin and should repair the skin $\&$ should retain the moisture for a long time. The moisture level and emolliency of skin can be measured in various methods such as electric conductance, trans epidermal water loss, Fourier transform infrared spectroscopy, photothermal imaging and confocal Raman spectroscopy [2-4]. In these methods measuring moisture level of skin is simple and reliable method to understand the variations in skin hydration \& emolliency.
In the present study we have validated the efficacy of the Verdura intense moisturizing lotion by two methods.

1. Moisture level

2. Dermascope images

Moisture levels will give understanding of the moisturization and retention and dermascopic study shows the repairing ability of the product. Results are presented in the paper.

\section{MATERIALS AND METHODS}

\section{Details of investigational product}

Verdura intense moisturizing lotion is a product of Dr. JRK's Research and Pharmaceuticals Pvt. Ltd., Chennai, India. It is formulated with Aloe vera extracts in a rich base to provide intense and long retention of moisturization.

\section{Clinical evaluation}

Study design

Ten volunteers were selected for the study. For analysis volunteers of both male and female in age

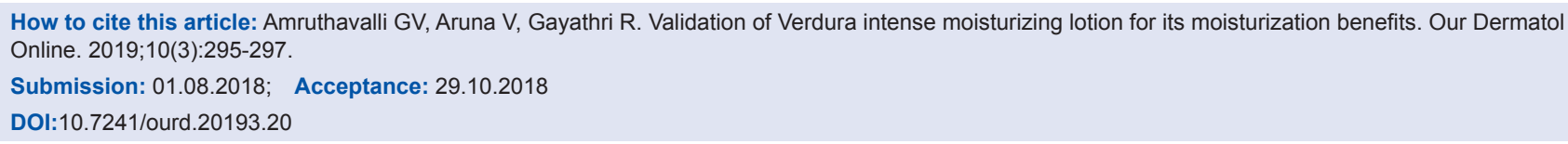


group of 20-45 was considered. Biceps region was selected as wear and tear is less and initial moisture levels were measured and marked these areas for further analysis.

Skin moisture level percentage was measured in 10 volunteers using skin analyzer. (PEPECARE Body Skin Facial Tester Oil Water Moisture Meter LCD Display Monitor Analyzer).

Study was planned for 5 consecutive days in following stages.

1. Initial moisturizing levels was measured - untreated

2. Test product was applied and left for 2-3 minutes and moisturizing levels was measured at 1 hour, 3 hour and 5 hours for all the volunteers

3. Dermascopic image was taken for all the patients as untreated, treated ( $3 \mathrm{~min}$ ) and treated 1 hour to understand the repairing ability

The test sample was applied as $1 \mathrm{mg} / \mathrm{cm}^{2}$ concentration on marked area. Moisture level was measured for all the volunteers in the time intervals of $3 \mathrm{~min}, 1$ hour, 3 hours and 5 hours respectively. Readings were noted.

\section{RESULTS}

The table 1 shows the mean moisture levels of 10 volunteers evaluated for 5 days. There is improvement in the moisture levels of the skin in 5 days treatment.

Table 1: Mean moisture levels in 5 day's treatment

\begin{tabular}{lccccc}
\hline & Untreated & $\begin{array}{c}\text { Treated } \\
\mathbf{3} \mathbf{~} \mathbf{m i n}\end{array}$ & $\begin{array}{c}\text { Treated } \\
\mathbf{1} \mathbf{~ h r}\end{array}$ & $\begin{array}{c}\text { Treated } \\
\mathbf{3} \mathbf{~} \mathbf{r}\end{array}$ & $\begin{array}{c}\text { Treated } \\
\mathbf{5} \mathbf{~ h r}\end{array}$ \\
\hline Day 1 & $29 \pm 4$ & $36 \pm 5$ & $33 \pm 2$ & $32 \pm 3$ & $29 \pm 3$ \\
Day 2 & $32 \pm 2$ & $41 \pm 2$ & $35 \pm 2$ & $32 \pm 2$ & $31 \pm 3$ \\
Day 3 & $32 \pm 2$ & $40 \pm 2$ & $36 \pm 2$ & $34 \pm 2$ & $31 \pm 2$ \\
Day 4 & $31 \pm 3$ & $55 \pm 7$ & $37 \pm 3$ & $35 \pm 3$ & $33 \pm 4$ \\
Day 5 & $32 \pm 2$ & $42 \pm 2$ & $37 \pm 2$ & $35 \pm 4$ & $33 \pm 2$ \\
\hline
\end{tabular}

Table 2: Summary of statistical data

\begin{tabular}{lcccccc}
\hline & \multicolumn{7}{c}{ Treatments } \\
\cline { 2 - 7 } & $\mathbf{1}$ day & 2 day & 3 day & 4 day & $\mathbf{5}$ day & Total \\
\hline $\mathrm{N}$ & 5 & 5 & 5 & 5 & 5 & 25 \\
$\sum \mathrm{X}$ & 156 & 214 & 178 & 168 & 157 & 873 \\
Mean & 31.2 & 42.8 & 35.6 & 33.6 & 31.4 & 34.92 \\
$\sum \mathrm{X}^{2}$ & 4874 & 9366 & 6348 & 5654 & 4941 & 31183 \\
Standard deviation & 1.3038 & 7.1903 & 1.6733 & 1.5166 & 1.6733 & 5.3923 \\
\hline \multicolumn{7}{c}{ Result dtails } \\
\hline Source & $\mathrm{SS}$ & $\mathbf{d f}$ & MS \\
\hline Between-treatments & 452.64 & 4 & 113.16 & $F=9.23002$ \\
Within-treatments & 245.2 & 20 & 12.26 & & \\
Total & 697.84 & 24 \\
\hline
\end{tabular}

Table 2 shows the f-ratio value is 9.23002 . The p-value is 0.000215 . The result is significant at $p<0.05$. Table 3 shows the dermascopic images of the treated and untreated areas for 5 consecutive days. There is an improvement in skin texture was observed.

\section{DISCUSSION}

Stratum corneum is the outermost layer of the epidermis. It protects the living cells beneath it by providing a tough barrier between the environment and the lower layers of the skin.

The normal functioning of the SC, however, can be disturbed in dry, flaky skin conditions. Moisturizers and emollients are required during these conditions.

In the present study, moisturization benefits was validated for verdura intense moisturizing lotion by measuring moisturizing levels on repeat treatment and the skin structure. Skin structure especially we to see the repair of the SC cells.

Table 3: Dermascope images untreated, treated $3 \mathrm{~min}$ and 1 hour

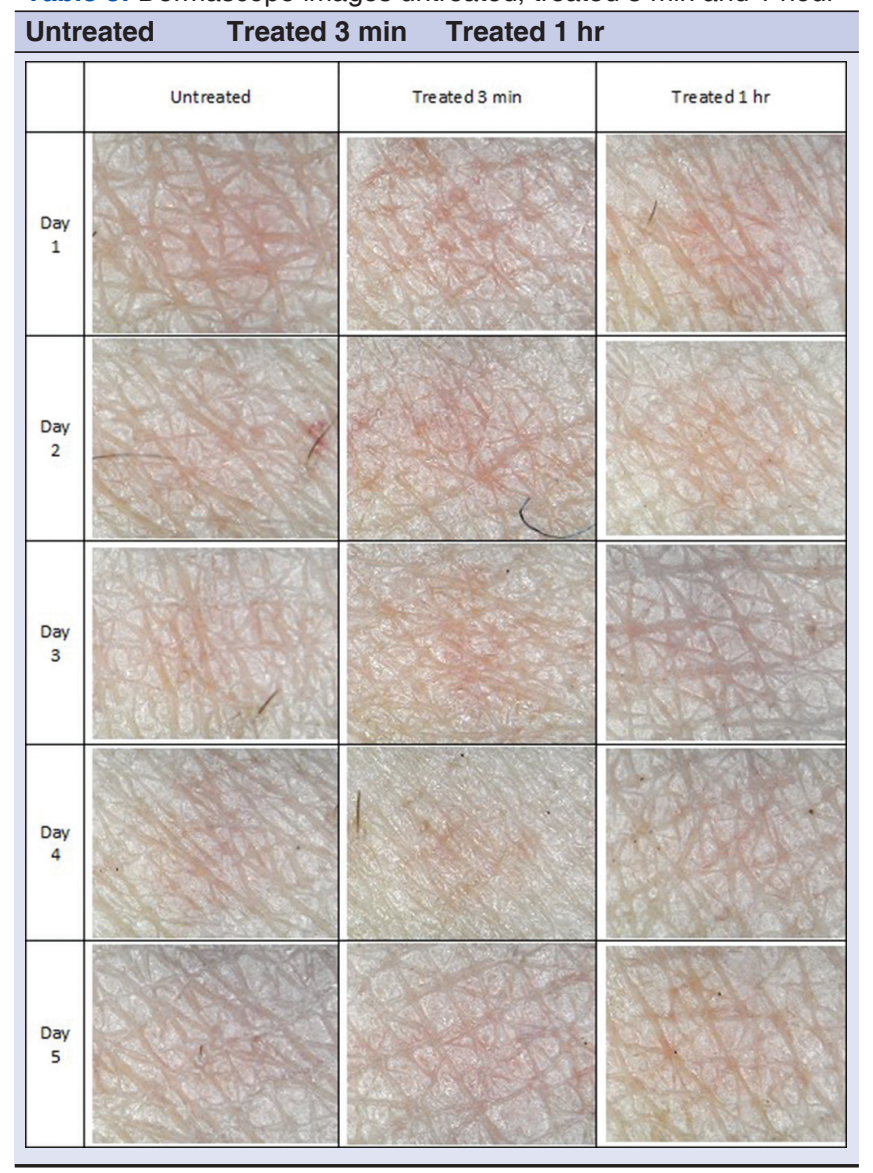


The moisture analysis conducted for 5 days at untreated zone i.e, before application of lotion at $3 \mathrm{~min}, 1 \mathrm{hr}$, 3 hours and 5 hr shows a gradual improvement of moisture levels from $29 \%$ to $32 \%$ with a standard deviation of 4 and 2 respectively.

In the treated region, at 3 min time also there is sudden raise in moisture levels due to application of lotion. The dermascope image also showed that there is constriction of cells after application of lotion. After an hour there is expansion of cells observed which shows the repair activity of cells. at the same time there is reduction in moisture levels compared to $3 \mathrm{~min}$ but more than the untreated i.e $36 \%$ ( 3 min on $1^{\text {st }}$ day) and $42 \%$ ( 3 min on $5^{\text {th }}$ day).

The moisture levels are $33 \%$ on day 1 ( 1 hour reading) and $37 \%$ on day 5 . The similar moisture levels are observed on 3 hour readings i.e $32 \%$ (day 1 ) and $35 \%$ on day 5 .

However, there is reduction in moisture levels by 5 hours but by day 5 there is a significant improvement which shows the repair of dry skin. The moisture levels on day 1 is $29 \%$ and improved to 33 by day 5 .

\section{CONCLUSION}

This study shows that there is improvement in the moisture levels of the skin with regular use of Verdura intense moisturizing lotion and also it repairs the damaged dry skin. The damage repair can be observed by reduction in the width of the lines between the cells. This reduction shows the improvement in the texture of skin. Thus verdura intense moisturizing lotion can be used for intense moisturization and damage repair of dry skin.

\section{Consent}

The examination of the patient was conducted according to the Declaration of Helsinki principles.

\section{REFERENCES}

1. Rawlings AV, Harding CR. Moisturization and skin barrier function. Dermatol Ther. 2004;17 Suppl 1:43-8.

2. Tagami H, Ohi M, Iwatsuki K, Kanamaru Y, Yamada M, Ichijo B. Evaluation of the skin surface hydration in vivo by electrical measurement. J Invest Dermatol, 1980;75:500-7.

3. Blank IH. Factors which influence the water content of the stratum corneum. J Invest Dermatol. 1952;18:433-40.

4. Suh EJ, Woo YA, Kim HJ. Determination of water content in skin by using a FT near infrared spectrometer. Arch Pham Res. 2005;28:458-62.

Copyright by. Gatla Venkata Amruthavalli, et al. This is an open-access article distributed under the terms of the Creative Commons Attribution License, which permits unrestricted use, distribution, and reproduction in any medium, provided the original author and source are credited.

Source of Support: Nil, Conflict of Interest: None declared. 